\title{
Effect of marine n-3 fatty acids on circulating inflammatory markers in healthy subjects and subjects with cardiovascular risk factors
}

\author{
Mari C. W. Myhrstad • Kjetil Retterstøl • \\ Vibeke H. Telle-Hansen - Inger Ottestad • \\ Bente Halvorsen · Kirsten B. Holven • Stine M. Ulven
}

Received: 14 June 2010/Revised: 29 September 2010/Accepted: 14 December 2010/Published online: 13 January 2011

(C) The Author(s) 2011. This article is published with open access at Springerlink.com

\begin{abstract}
Objective The aim of the present paper was to review the literature in order to summarize the effects of marine n-3 fatty acids on circulating inflammatory markers among healthy subjects, subjects with high risk of developing cardiovascular disease (CVD) and in patients with CVD in human intervention studies.

Methods A systematic literature search in PubMed was performed. Intervention studies describing the effects of marine n-3 fatty acids on circulating inflammatory markers in healthy subjects, subjects with high risk of CVD and patients with CVD were included. The following exclusion criteria were used: (1) interventions assessing inflammatory markers with ex vivo methods (2) interventions with children (3) articles describing animal or cell culture studies.
\end{abstract}

Responsible Editor: Claudia Kasserra.

M. C. W. Myhrstad · V. H. Telle-Hansen · I. Ottestad

S. M. Ulven $(\square)$

Faculty of Health, Nutrition and Management,

Akershus University College, PO box 423,

2001 Lillestrøm, Norway

e-mail: StineMarie.Ulven@hiak.no

K. Retterst $\varnothing 1$

Lipid Clinic, Oslo University Hospital, Rikshospitalet,

0027 Oslo, Norway

K. Retterstøl

The Norwegian Medicines Agency, 0950 Oslo, Norway

B. Halvorsen

Research Institute for Internal Medicine, Oslo University

Hospital, Rikshospitalet, 0027 Oslo, Norway

V. H. Telle-Hansen · I. Ottestad · K. B. Holven

Department of Nutrition, Institute of Basic Medical sciences,

University of Oslo, PO Box 1046, Blindern 0316 Oslo, Norway
Twenty-two articles were included. Additionally, 13 papers from their literature lists were included based on the same inclusion and exclusion criteria as the literature search. Results and conclusion Intervention studies with marine n-3 fatty acids administered from either fish or fish oil demonstrate different results on inflammatory markers. No firm conclusion can be drawn about the effect of marine n-3 fatty acids on circulating inflammatory markers in healthy individuals, individuals with high risk of developing CVD or individuals with CVD related diseases.

Keywords Omega-3 fatty acids - Atherosclerosis . Inflammation · Circulating inflammatory markers . PBMCs

\section{Introduction}

Fish consumption reduces the risk of developing cardiovascular disease (CVD) and CVD mortality [1,2]. Reduced total mortality and major coronary events, including fatal and non-fatal MI, are observed in intervention trials after intake of fish and fish oil containing the marine n-3 fatty acids eicosapentaenoic acid (EPA, 20:5 n-3) and docosahexaenoic acid (DHA, 22:6 n-3) [3-6]. The association between high intakes of marine n-3 fatty acids and decreased morbidity and mortality from CVD can be explained by the decrease in plasma triglycerides [7, 8], moderate reduction in blood pressure [9] and reduced blood plate aggregation [10,11]. Protection against cardiac arrhythmias has also been shown but the effect is still under discussion [12, 13].

Atherosclerosis is the underlying cause of most CVD. In atherosclerosis, the combined action of risk factors causes the gradual thickening of the arterial wall due to lipid 
accumulation to form an atherosclerotic plaque which can abruptly rupture, causing thrombosis. Inflammation has emerged as a major player in the development and progression of atherosclerosis [14]. There is an association between various biomarkers of inflammation and prospective CVD risk in apparently healthy individuals as well as in patients with CVD or heart failure. Systemic biomarkers of early and late atherosclerosis are of great clinical interest due to their potential for identifying high risk patients. Even though there are some good candidates, only C-reactive protein (CRP) has emerged as a leading biomarker of inflammation for clinical application $[15,16]$; other markers with appropriate robustness, sensitivity and specificity have not yet emerged. Based on the fact that endothelial dysfunction is the earliest manifestation of atherosclerosis, along with the involvement of oxidative stress and inflammation at all stages of coronary atherosclerosis development, biomarkers such as soluble intracellular adhesion molecule (sICAM-1) and soluble vascular adhesion molecule (sVCAM-1), soluble E-selectin (sE-sel) (for endothelial activation), interleukin (IL)-6 (transcriptional driver of CRP) and monocyte chemoattractant protein (MCP)-1 (produced by activated vasculature) are of particular interest (Table 1). So far CRP, IL-6, sVCAM-1 and sICAM-1 provide prognostic information beyond that obtained by clinical variables after acute coronary syndromes; these mediators seem to be powerful predictors of subsequent cardiovascular events.
[17, 18]. Recently, intake of marine n-3 fatty acids has been associated with reduced plasma levels of inflammatory markers [19-21].

The aim of the present paper was to review the literature in order to summarize the effects of marine n-3 fatty acids on circulating inflammatory markers among healthy subjects, subjects with high risk of developing CVD and in patients with CVD in human intervention studies.

\section{Methods of this review}

The systematic literature search was conducted in PubMed in 2009 using the following terms: "eicosapentaenoic acid OR docosapentaenoic acid OR docosahexaenoic acid OR omega-3 OR fish oil OR cod liver oil AND inflammation". The following limitations were included in the search: "added to PubMed in the last 10 years, published in the last 10 years, Humans, Clinical Trial, English". In total, 91 articles were identified. Based on these papers, we included all studies that measured the effect of marine $n-3$ fatty acids on circulating inflammatory markers in plasma or serum among apparently healthy individuals (Table 2), individuals with high risk of developing CVD (Table 3) or individuals with CVD (Table 4). Interventions with marine $\mathrm{n}-3$ fatty acids given as supplements or in the diet as fish were included. The following exclusion criteria were used: (1) interventions assessing inflammatory markers with ex

Table 1 Relevant inflammatory markers and their biological function

\begin{tabular}{|c|c|c|}
\hline Inflammatory markers & Abbreviation & Function \\
\hline \multicolumn{3}{|l|}{ Acute-phase protein } \\
\hline C-reactive protein & CRP & $\begin{array}{l}\text { CRP is associated with the formation of cytokines, chemokines } \\
\text { and the acute-phase response }\end{array}$ \\
\hline \multicolumn{3}{|l|}{ Cytokines } \\
\hline Interleukin-6 & IL-6 & $\begin{array}{l}\text { Induces acute-phase response (by inducing CRP), anti-body } \\
\text { secretion and differentiation }\end{array}$ \\
\hline Interleukin- $1 \alpha$, Interleukin- $1 \beta$ & IL- $1 \alpha$, IL- $1 \beta$ & $\begin{array}{l}\text { Proliferation and maturation of lymphocytes, involved in } \\
\text { inflammation and acute-phase response }\end{array}$ \\
\hline Interleukin-18 & IL-18 & Involved in the formation of Th1 cells \\
\hline Tumor necrosis factor- $\alpha$ is a cytokine & TNF- $\alpha$ & $\begin{array}{l}\text { Induces adhesion molecules- and cytokine expression, } \\
\text { involved in cell death }\end{array}$ \\
\hline \multicolumn{3}{|l|}{ Adhesion protein } \\
\hline Soluble intercellular adhesion molecule- 1 & sICAM-1 & Binds monocytes and lymphocytes to the endothelium \\
\hline Soluble vascular cell adhesion molecule- 1 & sVCAM-1 & Binds monocytes and lymphocytes to the endothelium \\
\hline sE-selectin & sE-sel & Recruits leukocytes to the inflammatory site \\
\hline sP-selectin & sP-sel & $\begin{array}{l}\text { Recruits leukocytes to the inflammatory site. Induces } \\
\text { monocytes and platelet interactions }\end{array}$ \\
\hline \multicolumn{3}{|l|}{ Chemokines } \\
\hline Monocyte chemoattractant protein-1 & MCP-1 & Facilitates migration of leukocytes to the intima \\
\hline Granulocyte-macrophage colony-stimulating factor & GM-CSF & Growth and differentiation of monocytes \\
\hline Interleukin-8 & IL-8 & Facilitates migration of leukocytes to the intima \\
\hline
\end{tabular}




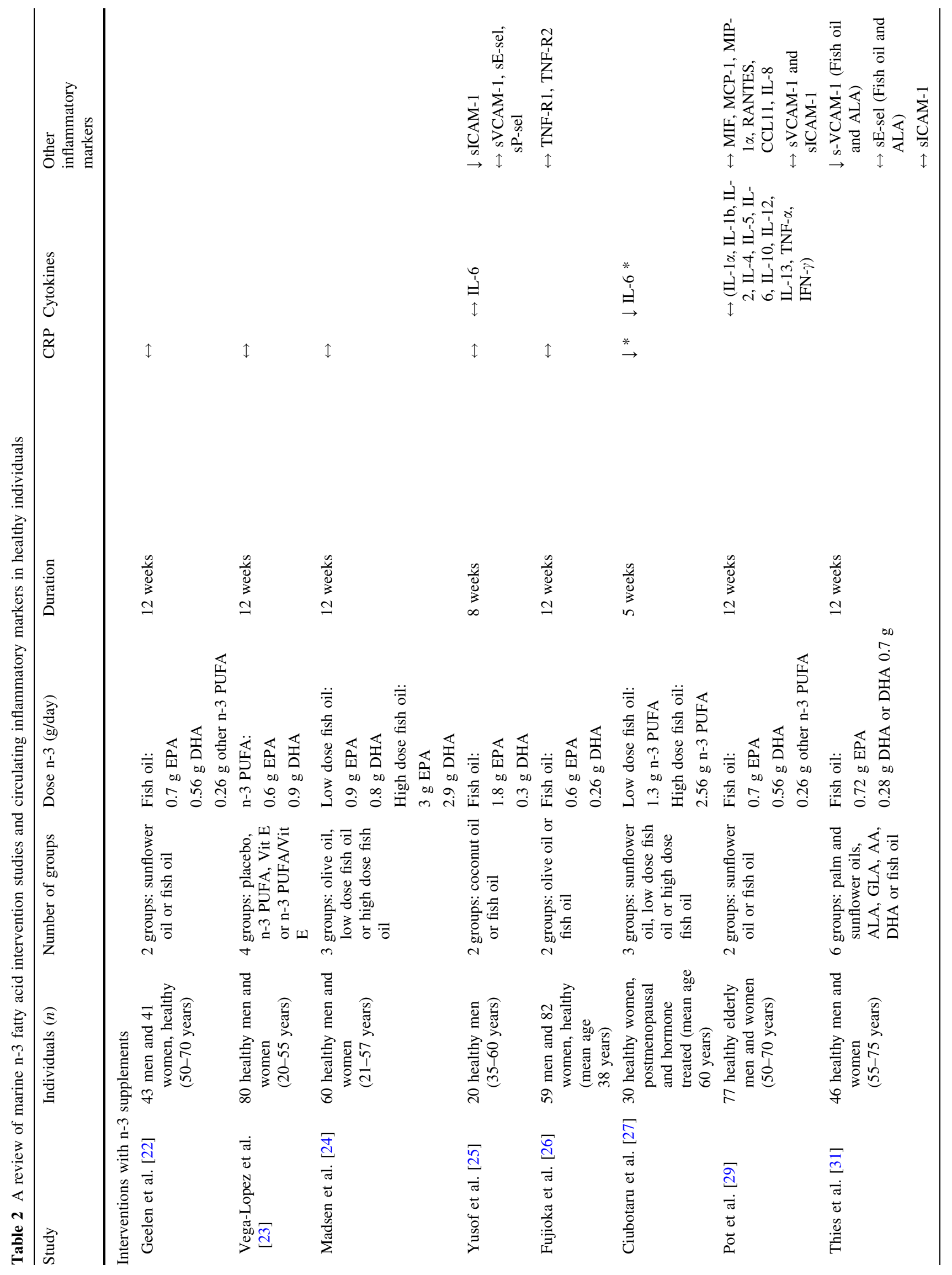




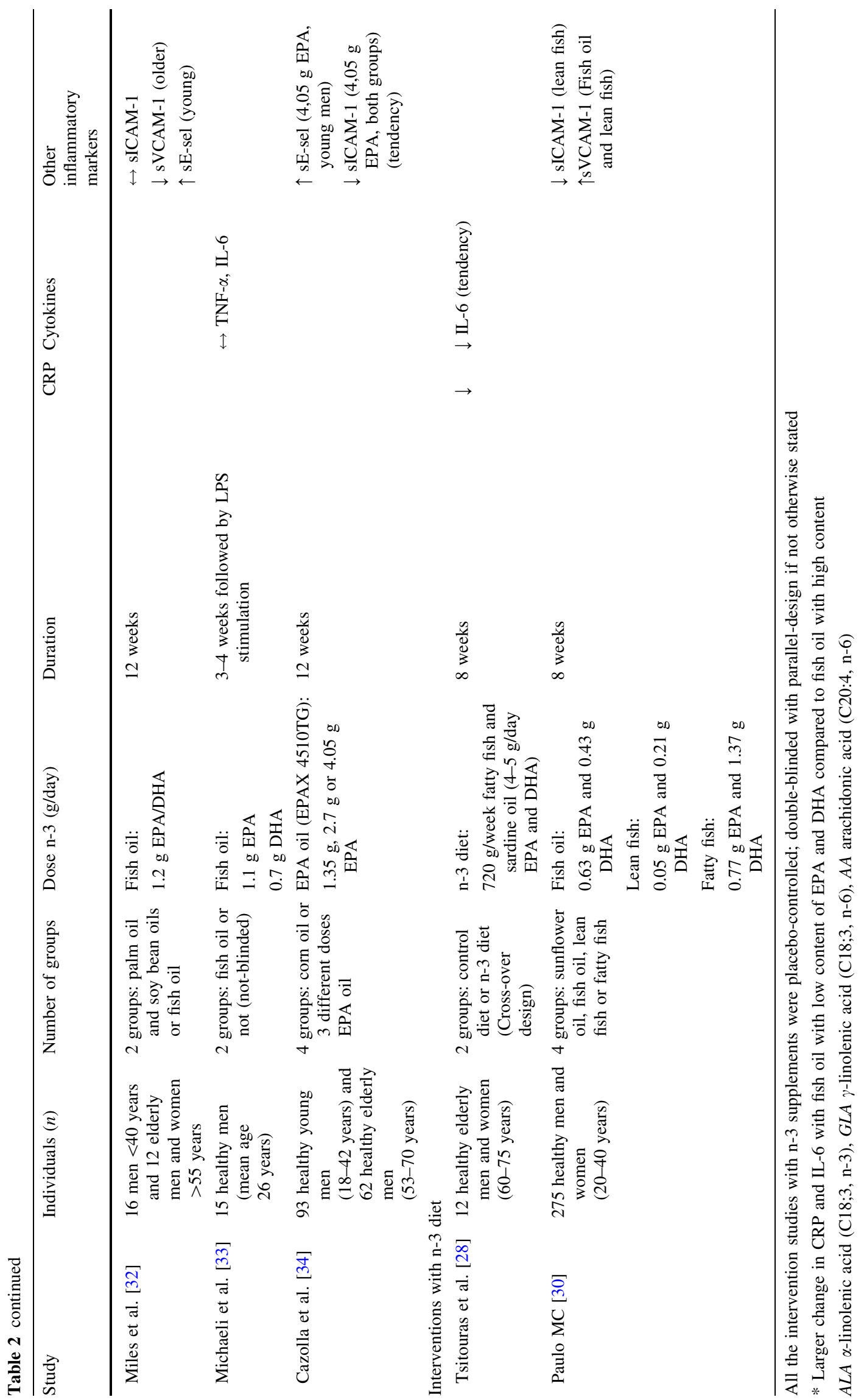




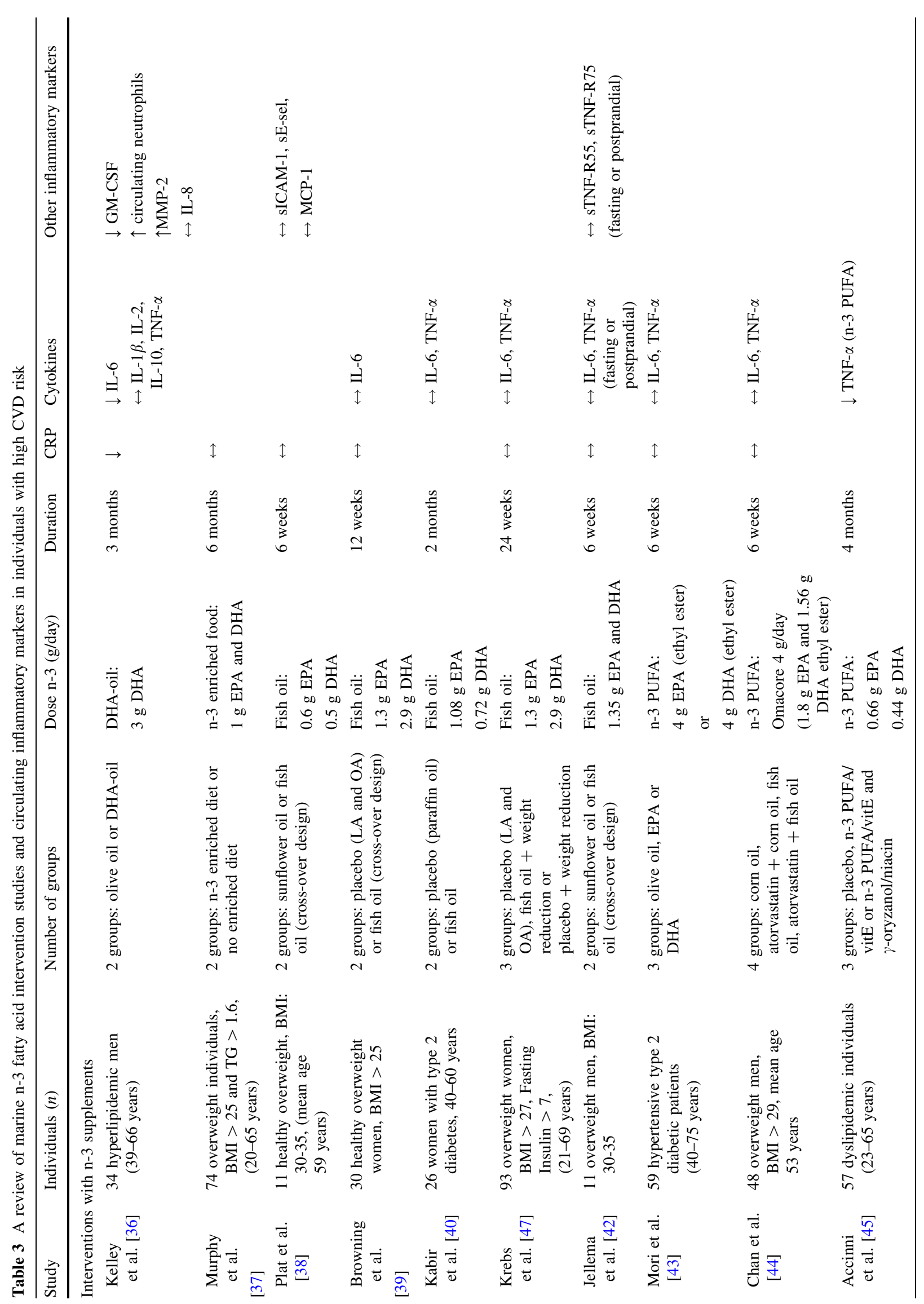




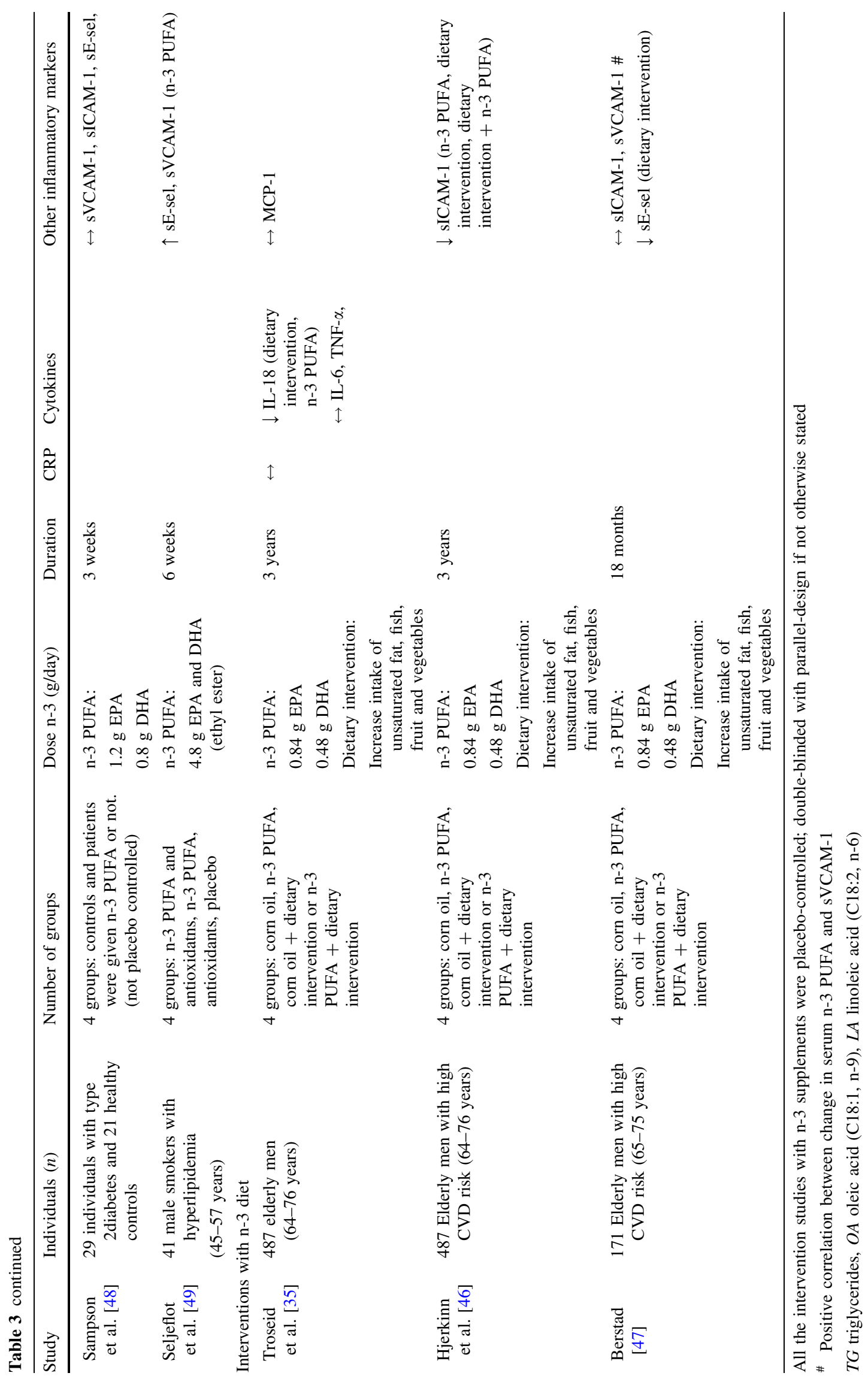




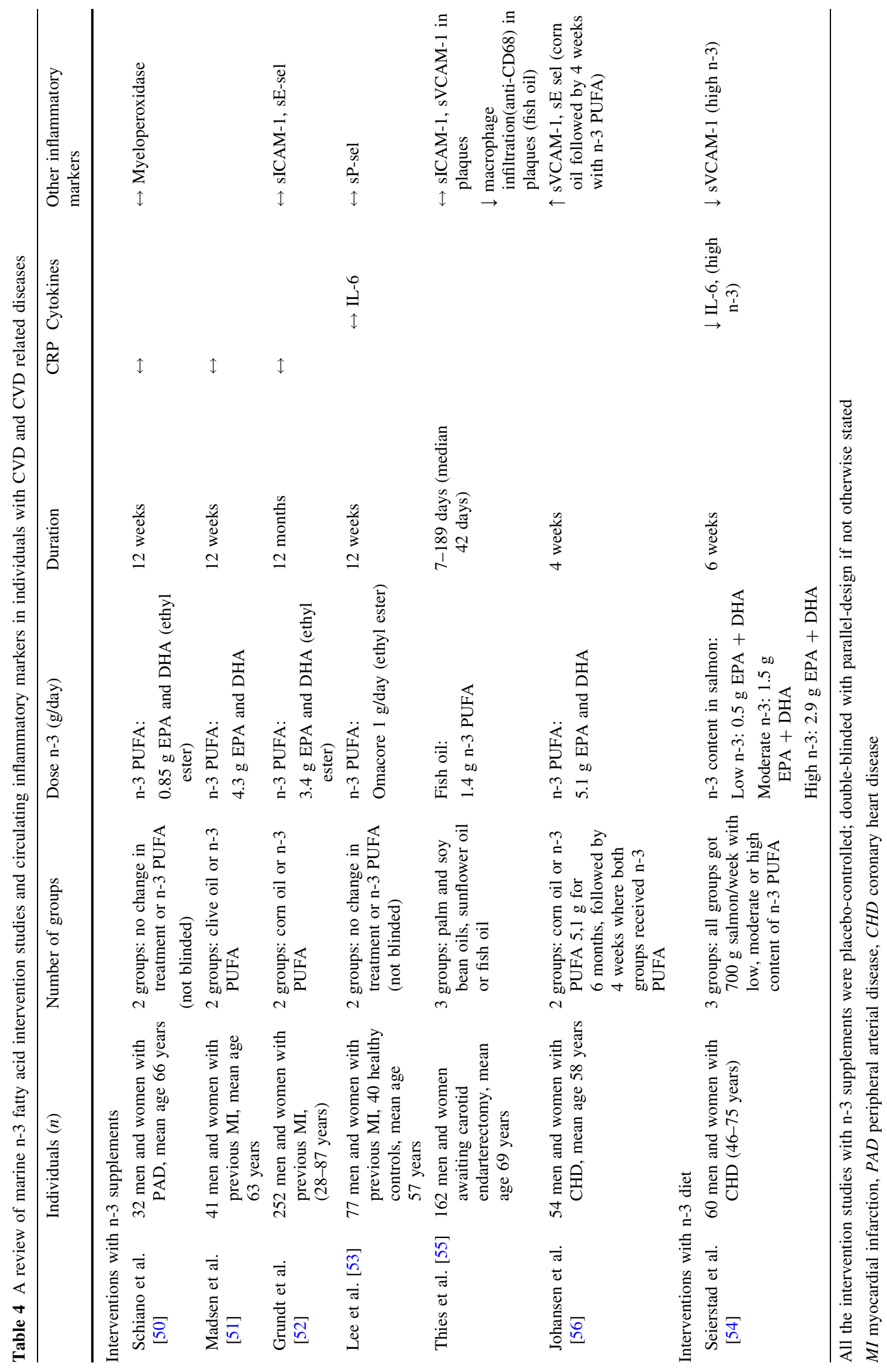


vivo methods (2), interventions with children (3) and articles describing animal or cell culture studies. After reading abstracts of the 91 papers, 22 articles were included. The literature lists of the selected papers were checked and 13 additional relevant papers were included based on the same inclusion and exclusion criteria as the literature search. The search was re-run in August 2010. However, no further papers were included from this search.

\section{Results}

Details of the 35 studies selected in this review are given in Tables 2, 3 and 4. Twenty-nine of these are based on marine n-3 fatty acids given as supplements, whereas six studies are dietary interventions with fish. Both categories of interventions are given in the tables.

The systematic review of the literature reveals that seven out of thirteen trials reported an effect on circulating inflammatory markers [22-34] in healthy individuals (Table 2). Six of the studies did not observe any changes in inflammatory markers [22-24, 26, 29, 33] (Table 2). C-reactive protein (CRP) was analyzed in seven of the trials, and in five of these no effect in CRP levels was observed [22-26], while a reduction in plasma concentration of CRP after intake of marine n-3 fatty acids was reported in two of the trials [27, 28]. Five of the thirteen trials analyzed the plasma concentration of IL-6. Two of these reported a reduction in IL-6 levels [27, 28], whereas three did not demonstrate any effect on IL-6 levels [25, 29, 33]. Table 2 also reveals that marine n-3 fatty acids reduced or had no effects on the concentration of SICAM-1 in five of the trials $[25,29,31,32,34]$. The concentration of sVCAM-1 was reduced, increased or unchanged after intake of marine $\mathrm{n}-3$ fatty acids among healthy individuals in these trials, whereas the level of sE-sel increased or remained unchanged [25, 31, 32, 34]. The effects of marine n-3 fatty acids on circulating inflammatory markers among individuals with a high risk of developing CVD are shown in Table 3 [35-49]. Four of fifteen trials reported a reduction in the concentration of inflammatory markers after intake of marine n-3 fatty acids [35, 36, 45, 46], while nine studies did not observe any effects on inflammation [37-44, 48]. An increase in inflammatory markers [49] was reported in one study. One of nine studies where CRP was analyzed demonstrated a reduction in the concentration of CRP after intake of marine n-3 fatty acids [36]. Three trials demonstrated a reduction in plasma cytokines or soluble adhesion molecules $[35,45,46]$. Table 4 reviews the effects of marine n-3 fatty acids on circulating inflammatory markers among individuals with CVD or CVD related diseases [50-56]. Five of seven studies showed no change in the concentration of inflammatory markers after intake of marine n-3 fatty acids [50-53,55], whereas one study reported a reduction in the levels of IL- 6 and sVCAM- 1 [54] and one trial showed an increase in the level of sE-sel and SVCAM -1 [56].

\section{Discussion and conclusion}

Effect of marine n-3 fatty acids on circulating inflammatory markers

Intervention trials with marine $\mathrm{n}-3$ fatty acids administered either from fish or fish oil supplements demonstrate different results on circulating inflammatory markers in healthy individuals, individuals with high risk of developing CVD or individuals with CVD related diseases. Based on these findings, a firm conclusion about the effect of marine n-3 fatty acids on circulating inflammatory markers cannot be drawn. The majority of the trials do not show any effect, however a decreased level of circulating CRP and IL- 6 are observed in some studies, whereas the circulating level of adhesion molecules inconsistently are elevated, decreased or not changed. The reasons for the different results may be several. First of all, a local inflammatory improvement in the arterial wall will not necessary be reflected in the circulation and may explain the lack of effect observed after intake of marine n-3 fatty acids. Several of the reported trials have a relative small number of study subjects and probably low statistical power. Variation in the results can also be explained by differences in the administration of the marine n- 3 fatty acids (supplement vs. diet) as well as the doses used. Furthermore, there is uncertainty regarding the background diet and to what extend the intervention changed the balance between n-3 and n- 6 fatty acids or altered the intake of saturated fat in the diet.

Comparing the results on the effects of marine n-3 fatty acids given as supplements on serum CRP in healthy individuals, the baseline level of CRP seems crucial. Ciubotaru et al. [27] reported a significant reduction in the level of CRP in postmenopausal women who had mean baseline levels of CRP above $6 \mathrm{mg} / \mathrm{L}$. This is in contrast to the results from Geelen et al. [22], Vega-Lopez et al. [23] and Madsen et al. [24] who did not observe any changes in the CRP serum levels. The baseline level of serum CRP in the subjects in these studies was approximately $1 \mathrm{mg} / \mathrm{L}$. Vega-Lopez et al. [23] failed to see any effect on the serum CRP level using a daily dose of $0.6 \mathrm{~g}$ EPA and 0.9 DHA for 12 weeks, which is in line with the results from Madsen et al. [24] who used two different daily doses $(3.0 \mathrm{~g}$ EPA +2.9 g DHA or 0.9 g EPA +0.8 g DHA). In contrast, Ciubotaru et al. [27] observed in postmenopausal women that the effect on CRP is dependent on the dose of 
marine n-3 fatty acids. A larger decrease in CRP was observed in the women who received the highest dose of marine $\mathrm{n}-3$ fatty acids $(2.56 \mathrm{~g} / \mathrm{d})$ compared to those receiving a daily dose of $1.3 \mathrm{~g}$ marine $\mathrm{n}-3$ fatty acids.

It is interesting to note that observed decrease in circulating CRP level was associated with decrease in circulating IL-6 level after intake of marine n-3 fatty acids, both via supplement or diet. In this review there are relatively few studies where marine n-3 fatty acids are administered as fish. When comparing these interventions with the interventions with fish oil, there are indications for a more prominent effect on circulating inflammatory markers with fish containing diet. Reductions or no change in one of the following markers (CRP, IL-6, sICAM-1, sVCAM-1, IL-18, sE-sel) are observed in all the six dietary studies included in this review. A possible explanation could be that subjects participating in dietary intervention trials may change their overall dietary pattern resulting in more awareness of healthy lifestyle, which again may influence the circulating inflammatory markers. Fish may also contain other bioactive compounds that may influence outcome.

New strategies for studying effects of marine n-3 fatty acids on inflammation

To further understand if marine n-3 fatty acids have an effect on inflammation in relation to atherosclerosis and the underlying molecular mechanisms responsible for their inflammatory response, new strategies should be considered. When inflammatory markers are measured in the circulation, the local effect in monocytes will not necessary be detected since the monocytes only contribute to about $5 \%$ of the total cells in plasma. The peripheral blood mononuclear cells (PBMCs) include monocytes and lymphocytes, which are cells central in inflammation and hence in the atherosclerosis process. The PBMCs are exposed to many of the same environmental factors as the arterial wall. Alterations in gene expression levels in these cells can thus be demonstrated early in the process before signs of inflammation can be seen in vivo, and thus constitute an important source of biomarkers for progression of atherosclerotic disease. PBMCs are easily available, which makes them suitable for studying gene expression of mediators involved in the early development of atherosclerosis [57]. A previous study recently demonstrated that 166 genes among 182 candidate cardiovascular genes were expressed in PBMCs [58].

The ability of marine n-3 fatty acids to alter gene expression has been clearly demonstrated in vitro and may account for its potential beneficial health effects. Fatty acids regulate expression of genes involved in lipid metabolism and inflammation by acting as ligands for the peroxisomal proliferator-activated receptors (PPARs) [59]. Fatty acids are also known to reduce the activation of the transcription factor nuclear factor kappa B $(\mathrm{NF}-\kappa \mathrm{B})$ probably by interference with the PPARs $[60,61]$ or the Tolllike receptors $[62,63]$. The $G$ protein-coupled receptor 120 (GPR120) has recently been characterized as an n-3 fatty acid receptor/sensor involved in the anti-inflammatory effects of n-3 fatty acids [64]. PPAR and PPAR target genes are expressed in PBMCs [65]. Dietary supplements containing fish oil have also been shown to affect expression of PI3K, Akt, NF- $\kappa \mathrm{B}$ and inflammatory cytokines in mononuclear cells [66].

To obtain a more comprehensive overview of the processes that are modulated by EPA and DHA, new "omics" technologies can be used to detect large scale changes in gene expression profiles (transcriptomics). Whole genome transcriptomic analysis in PBMCs will be valuable in further understanding the inflammatory role of n-3 fatty acids in humans. Recently, a human intervention study among elderly who daily consumed fish oil (containing $1.8 \mathrm{~g}$ EPA and DHA) for 26 weeks demonstrated changes in the gene expression profiles in PBMCs. Gene transcripts involved in inflammation and other processes of atherosclerosis were down-regulated among those who had consumed fish oil [67]. The combined use of other "omics" technologies, such as proteomics and metabolomics (lipidomics), in intervention studies will further extend the understanding of the effects of marine n-3 fatty acids on inflammation. Using proteomics technology, a recent study demonstrated that daily intake of $3.5 \mathrm{~g}$ fish oil down-regulated a number of proteins involved in the acute phase response and lipid/ lipoprotein metabolism [68]. Furthermore, Lankinen et al. [69] previously used a lipidomics approach in an intervention study where 33 individuals randomized to eat fatty fish (150 g four times per week), lean fish (150 g four times per week) or no fish, in 8 weeks. They observed a reduction in the level of lipid metabolites related to inflammation and insulin-signaling among those eating fatty fish.

No firm conclusion about the effect of marine n-3 fatty acids on circulating inflammatory markers can be drawn in this review. A systematic use of transcriptome, proteome, and metabolome technologies for the construction of network based models of biological processes has emerged as an exciting research approach in molecular biology and functional genomics. In future marine $\mathrm{n}-3$ supplementation and dietary intervention trials, systems biology combined with traditional circulating markers may help us to gain more insight into the underlying molecular mechanisms of marine $\mathrm{n}-3$ fatty acids responsible for the inflammatory response in the progression of atherosclerotic disease. 
Acknowledgments This work was supported by grants from the Norwegian Research Council, the Throne Holst Foundation and The Nordic Center of Excellence (NCoE) "SYSDIET" by Nordforsk.

Open Access This article is distributed under the terms of the Creative Commons Attribution Noncommercial License which permits any noncommercial use, distribution, and reproduction in any medium, provided the original author(s) and source are credited.

\section{References}

1. $\mathrm{Hu} \mathrm{FB}$, et al. Fish and omega-3 fatty acid intake and risk of coronary heart disease in women. JAMA. 2002;287(14):1815-21.

2. He K, et al. Accumulated evidence on fish consumption and coronary heart disease mortality: a meta-analysis of cohort studies. Circulation. 2004;109(22):2705-11.

3. Burr ML, et al. Effects of changes in fat, fish, and fibre intakes on death and myocardial reinfarction: diet and reinfarction trial (DART). Lancet. 1989;2(8666):757-61.

4. Dietary supplementation with $n-3$ polyunsaturated fatty acids and vitamin $\mathrm{E}$ after myocardial infarction: results of the GISSI-Prevenzione trial. Gruppo Italiano per lo Studio della Sopravvivenza nell'Infarto miocardico. Lancet. 1999;354(9177):447-55.

5. Tavazzi L, et al. Effect of n-3 polyunsaturated fatty acids in patients with chronic heart failure (the GISSI-HF trial): a randomised, double-blind, placebo-controlled trial. Lancet. 2008;372(9645):1223-30.

6. Yokoyama M, et al. Effects of eicosapentaenoic acid on major coronary events in hypercholesterolaemic patients (JELIS): a randomised open-label, blinded endpoint analysis. Lancet. 2007; 369(9567):1090-8.

7. Hartweg $\mathbf{J}$ et al. Omega-3 polyunsaturated fatty acids (PUFA) for type 2 diabetes mellitus. Cochrane Database Syst Rev. 2008;1:CD003205.

8. Harris WS. n-3 fatty acids and serum lipoproteins: human studies. Am J Clin Nutr. 1997;65(5 Suppl):1645S-54S.

9. Geleijnse JM, et al. Blood pressure response to fish oil supplementation: metaregression analysis of randomized trials. J Hypertens. 2002;20(8):1493-9.

10. Knapp HR. Dietary fatty acids in human thrombosis and hemostasis. Am J Clin Nutr. 1997;65(5 Suppl):1687S-98S.

11. Hornstra G. Influence of dietary fat type on arterial thrombosis tendency. J Nutr Health Aging. 2001;5(3):160-6.

12. Christensen JH, et al. n-3 polyunsaturated fatty acids, heart rate variability and ventricular arrhythmias in post-AMI-patients. A clinical controlled trial. Ugeskr Laeger. 1997;159(37):5525-9.

13. Nodari $S$, et al. The role of $n-3$ PUFAs in preventing the arrhythmic risk in patients with idiopathic dilated cardiomyopathy. Cardiovasc Drugs Ther. 2009;23(1):5-15.

14. Packard RR, Libby P. Inflammation in atherosclerosis: from vascular biology to biomarker discovery and risk prediction. Clin Chem. 2008;54(1):24-38.

15. Libby P, Ridker PM, Hansson GK. Inflammation in atherosclerosis: from pathophysiology to practice. J Am Coll Cardiol. 2009;54(23):2129-38

16. Vasan RS. Biomarkers of cardiovascular disease: molecular basis and practical considerations. Circulation. 2006;113(19):2335-62.

17. Postadzhiyan AS, et al. Circulating soluble adhesion molecules ICAM-1 and VCAM-1 and their association with clinical outcome, troponin $\mathrm{T}$ and C-reactive protein in patients with acute coronary syndromes. Clin Biochem. 2008;41(3):126-33.

18. Hartford $M$, et al. C-reactive protein, interleukin-6, secretory phospholipase A2 group IIA and intercellular adhesion molecule-
1 in the prediction of late outcome events after acute coronary syndromes. J Intern Med. 2007;262(5):526-36.

19. Calder PC. n-3 polyunsaturated fatty acids, inflammation, and inflammatory diseases. Am J Clin Nutr. 2006;83(6 Suppl):1505S$19 \mathrm{~S}$.

20. Lopez-Garcia E, et al. Consumption of (n-3) fatty acids is related to plasma biomarkers of inflammation and endothelial activation in women. J Nutr. 2004;134(7):1806-11.

21. Madsen T, et al. C-reactive protein, dietary n-3 fatty acids, and the extent of coronary artery disease. Am J Cardiol. 2001; 88(10):1139-42.

22. Geelen $A$, et al. Intake of n-3 fatty acids from fish does not lower serum concentrations of C-reactive protein in healthy subjects. Eur J Clin Nutr. 2004;58(10):1440-2.

23. Vega-Lopez S, et al. Supplementation with omega3 polyunsaturated fatty acids and all-rac alpha-tocopherol alone and in combination failed to exert an anti-inflammatory effect in human volunteers. Metabolism. 2004;53(2):236-40.

24. Madsen T, et al. The effect of dietary n-3 fatty acids on serum concentrations of C-reactive protein: a dose-response study. Br J Nutr. 2003;89(4):517-22.

25. Yusof HM, Miles EA, Calder P. Influence of very long-chain n-3 fatty acids on plasma markers of inflammation in middle-aged men. Prostaglandins Leukot Essent Fatty Acids. 2008;78(3):219-28.

26. Fujioka $\mathrm{S}$, et al. The effects of eicosapentaenoic acid-fortified food on inflammatory markers in healthy subjects-A randomized, placebo-controlled, double-blind study. J Nutr Sci Vitaminol (Tokyo). 2006;52(4):261-5.

27. Ciubotaru I, Lee YS, Wander RC. Dietary fish oil decreases C-reactive protein, interleukin-6, and triacylglycerol to HDLcholesterol ratio in postmenopausal women on HRT. J Nutr Biochem. 2003;14(9):513-21.

28. Tsitouras PD, et al. High omega-3 fat intake improves insulin sensitivity and reduces CRP and IL6, but does not affect other endocrine axes in healthy older adults. Horm Metab Res. 2008;40(3):199-205.

29. Pot GK, et al. No effect of fish oil supplementation on serum inflammatory markers and their interrelationships: a randomized controlled trial in healthy, middle-aged individuals. Eur J Clin Nutr. 2009;63(11):1353-9.

30. Paulo MC, et al. Influence of $n-3$ polyunsaturated fatty acids on soluble cellular adhesion molecules as biomarkers of cardiovascular risk in young healthy subjects. Nutr Metab Cardiovasc Dis. 2008;18(10):664-70.

31. Thies F, et al. Influence of dietary supplementation with long-chain $\mathrm{n}-3$ or n-6 polyunsaturated fatty acids on blood inflammatory cell populations and functions and on plasma soluble adhesion molecules in healthy adults. Lipids. 2001;36(11):1183-93.

32. Miles EA, et al. Influence of age and dietary fish oil on plasma soluble adhesion molecule concentrations. Clin Sci (Lond). 2001;100(1):91-100.

33. Michaeli B, et al. Effects of fish oil on the neuro-endocrine responses to an endotoxin challenge in healthy volunteers. Clin Nutr. 2007;26(1):70-7.

34. Cazzola R, et al. Age- and dose-dependent effects of an eicosapentaenoic acid-rich oil on cardiovascular risk factors in healthy male subjects. Atherosclerosis. 2007;193(1):159-67.

35. Troseid M, et al. Serum levels of interleukin-18 are reduced by diet and n-3 fatty acid intervention in elderly high-risk men. Metabolism. 2009;58(11):1543-9.

36. Kelley DS, et al. DHA supplementation decreases serum C-reactive protein and other markers of inflammation in hypertriglyceridemic men. J Nutr. 2009;139(3):495-501.

37. Murphy KJ, et al. Impact of foods enriched with n-3 long-chain polyunsaturated fatty acids on erythrocyte n-3 levels and cardiovascular risk factors. Br J Nutr. 2007;97(4):749-57. 
38. Plat J, et al. Weight loss, but not fish oil consumption, improves fasting and postprandial serum lipids, markers of endothelial function, and inflammatory signatures in moderately obese men. J Nutr. 2007;137(12):2635-40.

39. Browning LM, et al. The impact of long chain n-3 polyunsaturated fatty acid supplementation on inflammation, insulin sensitivity and CVD risk in a group of overweight women with an inflammatory phenotype. Diabetes Obes Metab. 2007;9(1):70-80.

40. Kabir M, et al. Treatment for 2 mo with n 3 polyunsaturated fatty acids reduces adiposity and some atherogenic factors but does not improve insulin sensitivity in women with type 2 diabetes: a randomized controlled study. Am J Clin Nutr. 2007;86(6): $1670-9$.

41. Krebs JD, et al. Additive benefits of long-chain n-3 polyunsaturated fatty acids and weight-loss in the management of cardiovascular disease risk in overweight hyperinsulinaemic women. Int J Obes (Lond). 2006;30(10):1535-44.

42. Jellema A, Plat J, Mensink RP. Weight reduction, but not a moderate intake of fish oil, lowers concentrations of inflammatory markers and PAI- 1 antigen in obese men during the fasting and postprandial state. Eur J Clin Invest. 2004;34(11):766-73.

43. Mori TA, et al. Effect of eicosapentaenoic acid and docosahexaenoic acid on oxidative stress and inflammatory markers in treated-hypertensive type 2 diabetic subjects. Free Radic Biol Med. 2003;35(7):772-81.

44. Chan DC, et al. Effect of atorvastatin and fish oil on plasma highsensitivity C-reactive protein concentrations in individuals with visceral obesity. Clin Chem. 2002;48(6 Pt 1):877-83.

45. Accinni R, et al. Effects of combined dietary supplementation on oxidative and inflammatory status in dyslipidemic subjects. Nutr Metab Cardiovasc Dis. 2006;16(2):121-7.

46. Hjerkinn EM, et al. Influence of long-term intervention with dietary counseling, long-chain n-3 fatty acid supplements, or both on circulating markers of endothelial activation in men with longstanding hyperlipidemia. Am J Clin Nutr. 2005;81(3):583-9.

47. Berstad P, et al. Supplementation with fish oil affects the association between very long-chain $n-3$ polyunsaturated fatty acids in serum non-esterified fatty acids and soluble vascular cell adhesion molecule-1. Clin Sci (Lond). 2003;105(1):13-20.

48. Sampson MJ, et al. n-3 polyunsaturated fatty acid supplementation, monocyte adhesion molecule expression and proinflammatory mediators in Type 2 diabetes mellitus. Diabet Med. 2001;18(1):51-8.

49. Seljeflot I, et al. Effects of omega-3 fatty acids and/or antioxidants on endothelial cell markers. Eur J Clin Invest. 1998; 28(8):629-35.

50. Schiano V, et al. Omega-3 polyunsaturated fatty acid in peripheral arterial disease: effect on lipid pattern, disease severity, inflammation profile, and endothelial function. Clin Nutr. 2008;27(2):241-7.

51. Madsen T, Christensen JH, Schmidt EB. C-reactive protein and n-3 fatty acids in patients with a previous myocardial infarction: a placebo-controlled randomized study. Eur J Nutr. 2007;46(7): 428-30.

52. Grundt $\mathrm{H}$, et al. Reduction in homocysteine by $n-3$ polyunsaturated fatty acids after 1 year in a randomised double-blind study following an acute myocardial infarction: no effect on endothelial adhesion properties. Pathophysiol Haemost Thromb. 2003;33(2): 88-95.
53. Lee KW, Blann AD, Lip GY. Effects of omega-3 polyunsaturated fatty acids on plasma indices of thrombogenesis and inflammation in patients post-myocardial infarction. Thromb Res. 2006; 118(3):305-12.

54. Seierstad SL, et al. Dietary intake of differently fed salmon; the influence on markers of human atherosclerosis. Eur J Clin Invest. 2005;35(1):52-9.

55. Thies $\mathrm{F}$, et al. Association of $\mathrm{n}-3$ polyunsaturated fatty acids with stability of atherosclerotic plaques: a randomised controlled trial. Lancet. 2003;361(9356):477-85.

56. Johansen $\mathrm{O}$, et al. The effect of supplementation with omega-3 fatty acids on soluble markers of endothelial function in patients with coronary heart disease. Arterioscler Thromb Vasc Biol. 1999;19(7):1681-6.

57. Pasterkamp G, Daemen M. Circulating cells: the biofactory for markers of atherosclerotic disease. Eur Heart J. 2008;29(22): 2701-2.

58. Visvikis-Siest $\mathrm{S}$, et al. Peripheral blood mononuclear cells (PBMCs): a possible model for studying cardiovascular biology systems. Clin Chem Lab Med. 2007;45(9):1154-68.

59. Lee $\mathrm{CH}$, Olson P, Evans RM. Minireview: lipid metabolism, metabolic diseases, and peroxisome proliferator-activated receptors. Endocrinology. 2003;144(6):2201-7.

60. Castrillo A, et al. Liver $X$ receptor-dependent repression of matrix metalloproteinase- 9 expression in macrophages. J Biol Chem. 2003;278(12):10443-9.

61. Joseph SB, et al. Reciprocal regulation of inflammation and lipid metabolism by liver X receptors. Nat Med. 2003;9(2):213-9.

62. Lee JY, et al. Differential modulation of Toll-like receptors by fatty acids: preferential inhibition by $n-3$ polyunsaturated fatty acids. J Lipid Res. 2003;44(3):479-86.

63. Wong SW, et al. Fatty acids modulate Toll-like receptor 4 activation through regulation of receptor dimerization and recruitment into lipid rafts in a reactive oxygen species-dependent manner. J Biol Chem. 2009;284(40):27384-92.

64. Oh da Y, et al. GPR120 is an omega-3 fatty acid receptor mediating potent anti-inflammatory and insulin-sensitizing effects. Cell. 2010;142(5):687-98.

65. Bouwens M, Afman LA, Muller M. Fasting induces changes in peripheral blood mononuclear cell gene expression profiles related to increases in fatty acid beta-oxidation: functional role of peroxisome proliferator activated receptor alpha in human peripheral blood mononuclear cells. Am J Clin Nutr. 2007;86(5):1515-23.

66. Weaver KL, et al. Effect of dietary fatty acids on inflammatory gene expression in healthy humans. J Biol Chem. 2009;284(23): 15400-7.

67. Bouwens M, et al. Fish-oil supplementation induces antiinflammatory gene expression profiles in human blood mononuclear cells. Am J Clin Nutr. 2009;90(2):415-24.

68. de Roos B, et al. Identification of potential serum biomarkers of inflammation and lipid modulation that are altered by fish oil supplementation in healthy volunteers. Proteomics. 2008;8(10): 1965-74.

69. Lankinen M, et al. Fatty fish intake decreases lipids related to inflammation and insulin signaling-a lipidomics approach. PLoS one. 2009;4(4):e5258. 